\title{
Corrigendum: Orthogonal gene knockout and activation with a catalytically active Cas9 nuclease
}

James E Dahlman, Omar O Abudayyeh, Julia Joung, Jonathan S Gootenberg, Feng Zhang \& Silvana Konermann

Nat. Biotechnol. 33, 1159-1161 (2015); published online 5 October 2015; corrected after print 4 February 2016

In the version of this article initially published, when discussing the data in Figure 2b, on p. 1160, we wrote, “...targeting the same $H B G 1 / 2$ promoter and found they had 32 and 55 perturbed transcripts...." This should have been " 31 and 55 perturbed transcripts" as in the sentence in the figure legend discussing the same data. The error has been corrected in the HTML and PDF versions of the article.

\section{Erratum: Phosphoproteomics takes it easy}

Paola Picotti

Nat. Biotechnol. 33, 929-930 (2015); published online 8 September 2015; corrected after print 4 February 2016

In the version of this article initially published, the page numbers in reference 1 were incorrect, and the reference thus linked online to the wrong article. The error has been corrected in the HTML and PDF versions of the article.

\section{Erratum: Resequencing 302 wild and cultivated accessions identifies genes related to domestication and improvement in soybean}

\footnotetext{
Zhengkui Zhou, Yu Jiang, Zheng Wang, Zhiheng Gou, Jun Lyu, Weiyu Li, Yanjun Yu, Liping Shu, Yingjun Zhao, Yanming Ma, Chao Fang, Yanting Shen, Tengfei Liu, Congcong Li, Qing Li, Mian Wu, Min Wang, Yunshuai Wu, Yang Dong, Wenting Wan, Xiao Wang, Zhaoli Ding, Yuedong Gao, Hui Xiang, Baoge Zhu, Suk-Ha Lee, Wen Wang \& Zhixi Tian Nat. Biotechnol. 33, 408-414 (2015); published online 2 February 2015; corrected after print 4 February 2016

In the version of this article initially published, grant no. 91131005 from the National Natural Science Foundation of China was inadvertently omitted. The error has been corrected in the HTML and PDF versions of the article.
} 